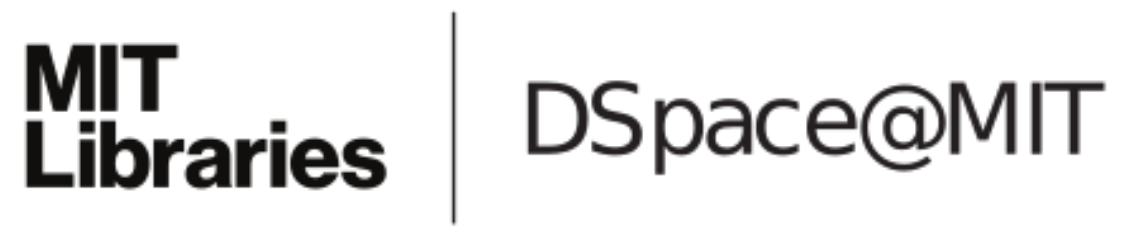

\author{
MIT Open Access Articles
}

Optimal Noise-Canceling Networks

The MIT Faculty has made this article openly available. Please share how this access benefits you. Your story matters.

Citation: Ronellenfitsch, Henrik, et al. "Optimal Noise-Canceling Networks." Physical Review Letters, vol. 121, no. 20, Nov. 2018. ( 2018 American Physical Society

As Published: http://dx.doi.org/10.1103/PhysRevLett.121.208301

Publisher: American Physical Society

Persistent URL: http://hdl.handle.net/1721.1/119241

Version: Final published version: final published article, as it appeared in a journal, conference proceedings, or other formally published context

Terms of Use: Article is made available in accordance with the publisher's policy and may be subject to US copyright law. Please refer to the publisher's site for terms of use. 


\title{
Optimal Noise-Canceling Networks
}

\author{
Henrik Ronellenfitsch, ${ }^{1, *}$ Jörn Dunkel, ${ }^{1, \dagger}$ and Michael Wilczek ${ }^{2, \$}$ \\ ${ }^{1}$ Department of Mathematics, Massachusetts Institute of Technology, Cambridge, Massachusetts 02139-4307, USA \\ ${ }^{2}$ Max Planck Institute for Dynamics and Self-Organization, 37077 Göttingen, Germany
}

(Received 22 July 2018; revised manuscript received 3 October 2018; published 16 November 2018)

\begin{abstract}
Natural and artificial networks, from the cerebral cortex to large-scale power grids, face the challenge of converting noisy inputs into robust signals. The input fluctuations often exhibit complex yet statistically reproducible correlations that reflect underlying internal or environmental processes such as synaptic noise or atmospheric turbulence. This raises the practically and biophysically relevant question of whether and how noise filtering can be hard wired directly into a network's architecture. By considering generic phase oscillator arrays under cost constraints, we explore here analytically and numerically the design, efficiency, and topology of noise-canceling networks. Specifically, we find that when the input fluctuations become more correlated in space or time, optimal network architectures become sparser and more hierarchically organized, resembling the vasculature in plants or animals. More broadly, our results provide concrete guiding principles for designing more robust and efficient power grids and sensor networks.
\end{abstract}

DOI: $10.1103 /$ PhysRevLett.121.208301

Fluctuations fundamentally limit the function and efficiency of physical [1] and biological [2,3] networks across a wide spectrum of scales. Important examples range from atmospheric turbulence [4,5] affecting large telescope arrays [6], wind farms [7-11], and power grids [12-16] to neuronal noise in the auditory $[17,18]$ and visual $[19,20]$ cortices, and extrinsic and intrinsic fluctuations [21] in gene expression pathways [22,23]. Over the last decades, remarkable progress has been made in the development and understanding of noise-suppression strategies [24,25] and their limits $[2,26]$ in physical $[11,27,28]$ and biological $[17,19,29]$ networks. Classical adaptive noise filtering [30-32] utilizes active control [33,34], and networks can be optimized for active controllability [35-37] and/or transport efficiency [38-43]. Still lacking at present are generic design principles for the construction of optimal passive noise-canceling networks (NCNs). While passive noise reduction has been demonstrated for single oscillators [44], it is not yet well understood how the architecture and efficiency of optimal NCNs depends on the input correlations and cost constraints in natural and man-made systems. Deciphering these dependencies can yield more robust sensory network and power grid designs and may also help clarify the role of noise-reduction in biological network evolution.

Correlated input fluctuations can have profound biomedical or technological consequences in hierarchical network structures. For instance, the detection neurons of the retina are subject to correlated fluctuations [45] which are passed on to the visual cortex where input noise has been shown to affect neural processing [19]. Similarly, deficient noise cancellation in dysfunctional auditory subnetworks has been proposed as a potential cause of tinnitus [17,18]. Another conceptually related problem of rapidly increasing importance is the feed-in of spatiotemporally correlated power fluctuations from solar and wind farms into multinational power grids [5,7,9,11-14,46-49]. These examples raise the general question of the extent to which efficient noise cancellation can be hard wired into a network's architecture if the signal fluctuations have known statistics.

Here, we show both analytically and numerically for generic oscillator networks [12,46,50-52] that it is indeed possible to design optimized weighted network topologies capable of suppressing "colored" fluctuations $[9,53]$ as typically present in biological and engineered systems. In stark contrast to the widely studied problem of optimal synchronization [27,54-63], our results imply that optimal NCNs harness desynchronization to reduce fluctuations globally. Importantly, NCNs operate purely passively, canceling out a substantial fraction of the input fluctuations without requiring active smoothing-the network itself acts as the filter. As a general principle, we find that the more correlated fluctuating inputs are in space or time, the sparser and the more hierarchically organized the NCN will be. Interestingly, the best-performing networks are often reminiscent of leaf venation or animal vasculature, supporting the view that robustness against fluctuations has been an evolutionary factor $[39,64]$. The mathematical analysis below thus provides detailed guidance for how to use biomimetic network topologies to improve noise robustness in engineered grids and sensor networks.

To investigate noise cancellation in a broadly applicable setting, we consider a generic model of $N$ spatially distributed, nonlinearly coupled second-order phase 

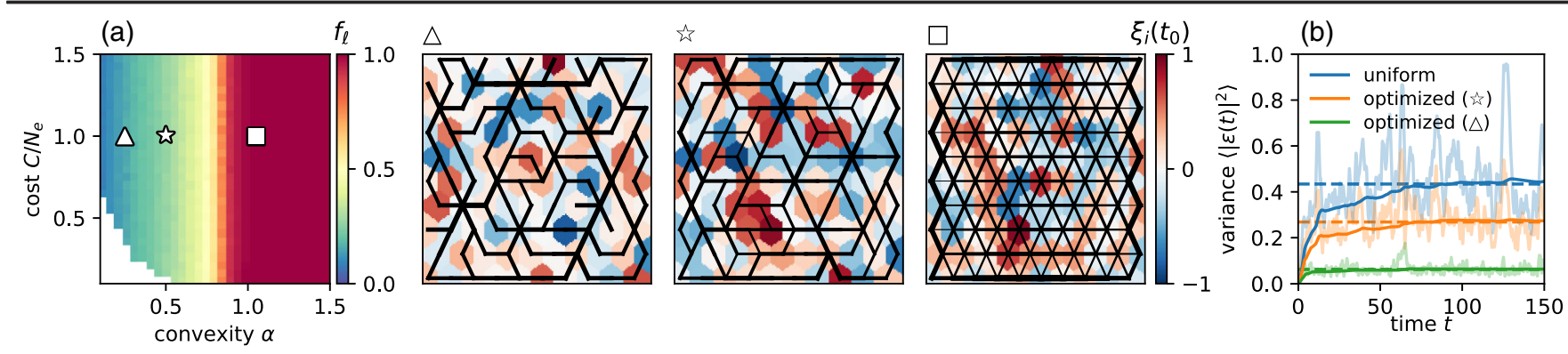

FIG. 1. Even for spatially incoherent white noise input $\sigma, \tau \rightarrow 0$, optimal NCNs exhibit a nontrivial sparse topology independent of the nonlinear steady state. (a) The fraction of loops $f_{\ell}=N_{\ell} / N_{\text {tri }}$, where $N_{\ell}$ is the number of loops in the optimized network and $N_{\text {tri }}$ is the number of loops in a triangular grid, measures the topology of optimal networks. Each of the $30 \times 30$ pixels in the cost-convexity phase diagram is an average over 15 optimal networks obtained for different uniformly random initial $B_{i j}$. In the white domain, no solutions to Eq. (2) were found. The NCN topology $f_{\ell}$ is effectively independent of $C$. Panels $\triangle$, $i$, $\square$ show examples of optimal NCNs with different sparsities, with edge thicknesses proportional to $B_{i j}^{\alpha}$. Backgrounds show one instance of the spatial feed-ins $\xi_{i}\left(t_{0}\right)$ normalized to $(-1,1)$. (b) Time-averaged variance $\left\langle|\boldsymbol{\varepsilon}(t)|^{2}\right\rangle$ and instantaneous variances $|\boldsymbol{\varepsilon}(t)|^{2}$ (faint) obtained from numerical solutions of Eq. (1) on uniform and optimized network topologies for $\alpha=0.25(\triangle)$ and $\alpha=0.5$ ( $\downarrow$ ) with edge cost $C=1$ and centered inputs. Analytically predicted variances (dashed) agree with the simulations.

oscillators, with phase angles $\delta_{i}(t)$ at each network node $i$, governed by

$$
\ddot{\delta}_{i}=-\gamma \dot{\delta}_{i}+\sum_{j=1}^{N} B_{i j} \sin \left(\delta_{i}-\delta_{j}\right)+P_{i}(t),
$$

where $\gamma$ is a damping coefficient. The oscillator couplings are symmetric, $B_{i j}=B_{j i}$, and $P_{i}(t)$ is the fluctuating net signal or power input at site $i$. Equation (1) has been successfully applied to describe the dynamics of power grids [50]. The Kuramoto model [51,54] is recovered in the overdamped limit, for which all subsequently derived results remain valid after a transformation of parameters (Supplemental Material [65]). The fluctuating inputs can be decomposed as $P_{i}(t)=\bar{P}_{i}+\xi_{i}(t)$, where $\xi_{i}(t)$ are the fluctuations around the constant mean $\bar{P}_{i}$. Because Eq. (1) is invariant under a constant shift $\delta_{i} \rightarrow \delta_{i}+c$, it is possible to split off the irrelevant dynamics of the mean $(1 / N) \sum_{j} \delta_{j}$ (Supplemental Material [65]). As a result, only the centered inputs $\bar{P}_{i}^{c}=\bar{P}_{i}-(1 / N) \sum_{j} \bar{P}_{j}$ and $\xi_{i}^{c}=\xi_{i}-(1 / N) \sum_{j} \xi_{j}$ are relevant. Adopting this mean-centered frame of reference from now on, we write $\delta_{i}(t)=\bar{\delta}_{i}+\varepsilon_{i}(t)$ for constant average phase angles $\bar{\delta}_{i}$ and fluctuations $\varepsilon_{i}(t)$. Assuming that the angle fluctuations $\varepsilon_{i}(t)$ are small and linearizing around $\bar{\delta}_{i}$, we obtain the coupled set of equations,

$$
\begin{aligned}
0 & =\sum_{j=1}^{N} B_{i j} \sin \left(\bar{\delta}_{i}-\bar{\delta}_{j}\right)+\bar{P}_{i}^{c} \\
\ddot{\varepsilon}_{i} & =-\gamma \dot{\varepsilon}_{i}+\sum_{j=1}^{N}\left[B_{i j} \cos \left(\bar{\delta}_{i}-\bar{\delta}_{j}\right)\right]\left(\varepsilon_{i}-\varepsilon_{j}\right)+\xi_{i}^{c}(t) .
\end{aligned}
$$

The zeros of the nonlinear algebraic Eq. (2) correspond to fixed points of Eq. (1). Our main goal here is to use Eq. (3) to derive and characterize optimal couplings $B_{i j}$ that minimize the total fluctuation variance $\left\langle|\boldsymbol{\varepsilon}(t)|^{2}\right\rangle$, where the vector $\boldsymbol{\varepsilon}(t)$ has components $\varepsilon_{i}(t)$, the total instantaneous variance is the norm $|\boldsymbol{\varepsilon}(t)|^{2}$, and $\langle\cdot\rangle$ denotes a time average. The optimal network connectivity $B_{i j}$ will depend on the statistics of the input fluctuations, encoded in the elements $R_{i j}\left(t, t^{\prime}\right)=\left\langle\xi_{i}(t) \xi_{j}\left(t^{\prime}\right)\right\rangle$ of the covariance matrix $R$.

Throughout, we assume that spatiotemporal correlations factorize, although the general approach extends to the nonfactorizing case. For the time correlations, we focus on colored Ornstein-Uhlenbeck noise [53] with $R\left(t, t^{\prime}\right)=\hat{R} e^{-\left|t-t^{\prime}\right| / \tau} /(2 \tau)$. In the limit of correlation time $\tau \rightarrow 0$, white noise is recovered with $R\left(t, t^{\prime}\right)=\hat{R} \delta\left(t-t^{\prime}\right)$. For the spatial part $\hat{R}=\left(\hat{R}_{i j}\right)$, we choose generic isotropic and homogeneous Gaussian covariances $\hat{R}_{i j}=e^{-\left|\mathbf{x}_{i}-\mathbf{x}_{j}\right|^{2} /\left(2 \sigma^{2}\right)}$, where $\mathbf{x}_{i}$ is the spatial position of oscillator $i$ and $\sigma$ is a correlation length. In the limit $\sigma \rightarrow 0$, the feed-ins become incoherent with $\hat{R}_{i j}=\delta_{i j}$. The total fluctuation variance $\left\langle|\boldsymbol{\varepsilon}(t)|^{2}\right\rangle$ can be calculated analytically for any $\hat{R}$ in the Langevin formalism (Supplemental Material [65]),

$$
\left\langle|\boldsymbol{\varepsilon}(t)|^{2}\right\rangle=\frac{1}{2 \gamma} \operatorname{tr}\left[\left(\mathbb{1}+\frac{\tau^{2}}{1+\gamma \tau} L\right)^{-1} L^{\dagger} \hat{R}\right],
$$

where $L$ is the weighted graph Laplacian matrix of the network with the weights of edge $(i j)$ given by $B_{i j} \cos \left(\bar{\delta}_{i}-\bar{\delta}_{j}\right)$, and tr is the matrix trace. The pseudoinverse $L^{\dagger}$ implicitly acts as a projection to center $\hat{R}$. In the white-noise limit $\tau \rightarrow 0$, Eq. (4) reduces to

$$
\left\langle|\boldsymbol{\varepsilon}(t)|^{2}\right\rangle=\frac{1}{2 \gamma} \operatorname{tr}\left(L^{\dagger} \hat{R}\right) .
$$

The structure of Eqs. (4) and (5) implies that, in principle, arbitrarily small variances $\left\langle|\boldsymbol{\varepsilon}(t)|^{2}\right\rangle$ can be achieved by choosing the $B_{i j}$ arbitrarily large. In natural or engineered 


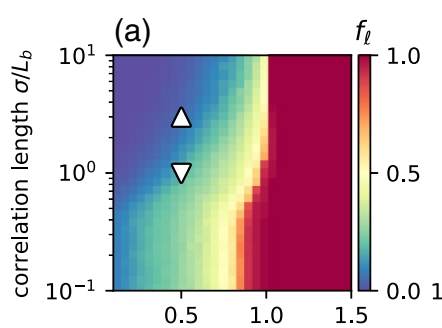

(b)
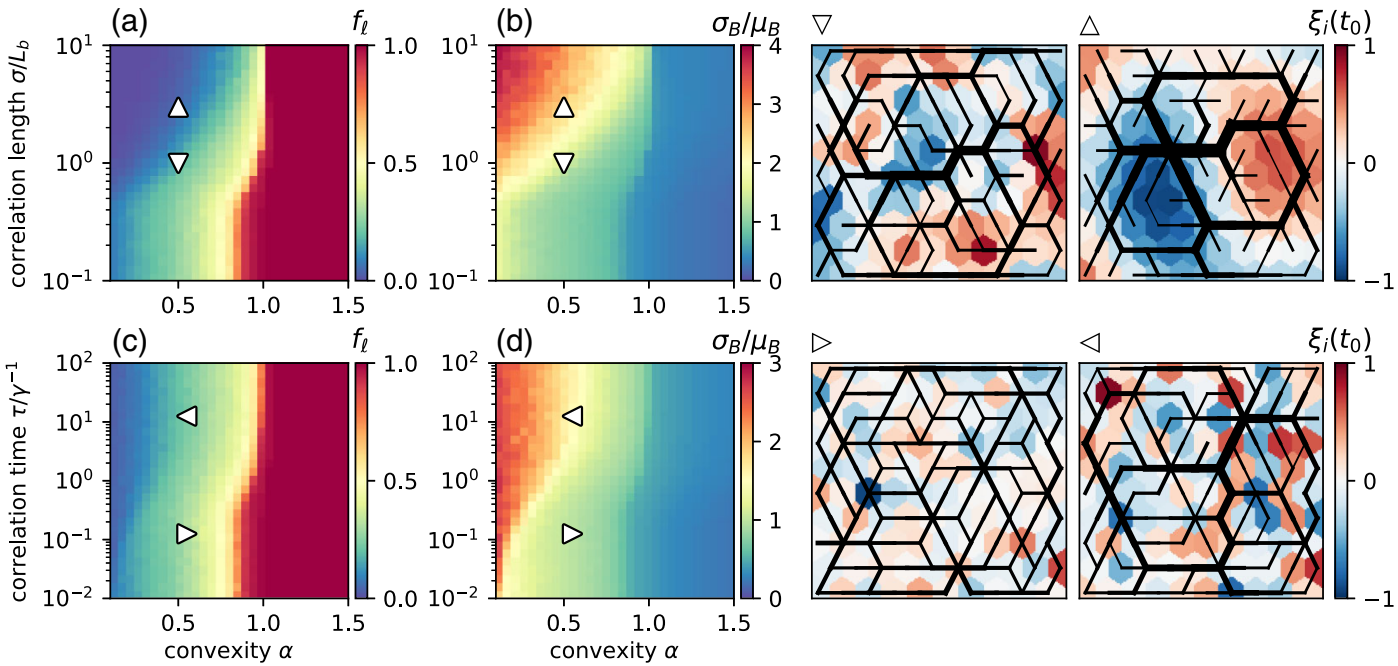

FIG. 2. Spatial and temporal input correlations lead to a similar hierarchical NCN organization despite acting through different mechanisms. (a),(b) Gaussian spatial correlations $\sigma>0$ with temporal white noise $\tau \rightarrow 0$. The loop fractions $f_{\ell}$ in (a) show that NCN topology depends largely on $\alpha$, although the transition between loopy and sparse phase shifts when the correlation scale $\sigma$ approaches the mean edge length $L_{b}$. For $\sigma \gg L_{b}$ networks become sparser when $\alpha \sim 1$. (b) The coupling variance $\sigma_{B}$, normalized by the mean $\mu_{B}$, indicates that nonuniform hierarchical patterns and sparsity are strongly correlated. (c),(d) Ornstein-Uhlenbeck colored noise $\tau>0$ with spatially incoherent feed-ins $\sigma \rightarrow 0$ shows hierarchical patterns similar to those in panels (a),(b). Examples of optimal networks at the positions marked by symbols in the phase diagrams illustrate the transitions from dense uniform networks to sparse hierarchical networks with increasing spatial or temporal correlation. Each of the $30 \times 30$ pixels in (a)-(d) is an average over 15 optimal networks.

real-world networks, however, the allowed values of the $B_{i j}$ are restricted by construction or maintenance costs. To account for this fact, we adopt here the widely used $[39,40,57,64,66]$ cost constraint $\sum_{(i j)} B_{i j}^{\alpha}=N_{e} C$, where $\alpha>0$ is a convexity parameter, $C$ the cost per edge, and $N_{e}$ the number of edges in the network. In the concave regime $\alpha<1$, one expects sparse networks because it becomes more economical to construct a single edge with a large coupling rather than to distribute over, say, two smaller ones. Since many natural networks are sparse, and sparsity is desirable in engineering, this concave range arguably comprises the most interesting part of phase space. The cost-constrained optimization is carried out starting from a given base network and initial $B_{i j}$. Optimal weights are found iteratively using the method of Lagrange multipliers (Supplemental Material [65]). Weights $B_{i j}=0$ in the final optimized network correspond to edges being pruned from the base network, and thus to changes in topology. In the case of white noise in time and close to synchrony $\left(\bar{\delta}_{i} \approx 0\right)$, the minima have an interesting interpretation: using the eigendecomposition $\hat{R}=\sum_{k} \rho_{k} \boldsymbol{r}_{k} \boldsymbol{r}_{k}^{\top}$, one finds the defining relation $\alpha \lambda B_{i j}^{\alpha+1}=\sum_{k} \rho_{k}\left[B_{i j}\left(\varepsilon_{i}^{(k)}-\varepsilon_{j}^{(k)}\right)\right]^{2}$, where the $\boldsymbol{\varepsilon}^{(k)}$ are steady-state angles in the presence of steady feed-ins $\boldsymbol{r}_{k}$. Thus, the optimal couplings are directly related to a weighted average over local steady state flows. In the general case, additional terms appear (Supplemental Material [65]). Armed with these analytical insights, we now turn to the numerical investigation of optimal NCNs for different input noise statistics.
As base networks, we explore planar triangular grids which are approximately realized in many biological and engineering systems such as cilia $[67,68]$ or staggered wind farms [69]. The number of nodes is $N=100$ and damping is fixed at $\gamma=0.5$, following Ref. [50]. The uncentered steady feed-ins are $\bar{P}_{i}=\eta_{i}$, where the $\eta_{i}$ are independent Gaussian random variables with zero mean and unit variance. Covariance matrices are normalized to $\operatorname{tr}(\hat{R})=1$, bringing steady state background and fluctuations to a similar scale. Numerical solutions of Eq. (1) were obtained using the Euler-Maruyama scheme with time step $\Delta t=10^{-3}$. All main results remain valid for other grid geometries (Supplemental Material [65]).

Already in the simplest case, when node inputs are white noise in time $(\tau \rightarrow 0)$ and spatially incoherent $(\sigma \rightarrow 0)$, optimal NCNs exhibit nontrivial topologies in the sparse regime $0<\alpha<1$ [Fig. 1]. The fraction of loops $f_{\ell}=N_{\ell} / N_{\text {tri }}$, where $N_{\ell}$ is the number of loops in the optimal network and $N_{\text {tri }}$ is the number of loops in the full triangular grid, decreases with $\alpha$ [Fig. 1(a)]. This indicates that optimal NCNs become sparser for $\alpha \rightarrow 0$. The nonzero couplings in the optimized network have similar magnitude for uncorrelated inputs [Fig. 1(a), $\triangle$, $\lesssim)$ ], and the optimal networks do not follow any symmetry of the base network. As expected, optimal networks become dense for $\alpha>1$ [Fig. 1(a),( $\square)]$ and retain the base network topology. The nonlinear steady state, despite being fully taken into account in our optimization procedure, has little influence on the structure of optimal NCNs. Decreasing the mean 


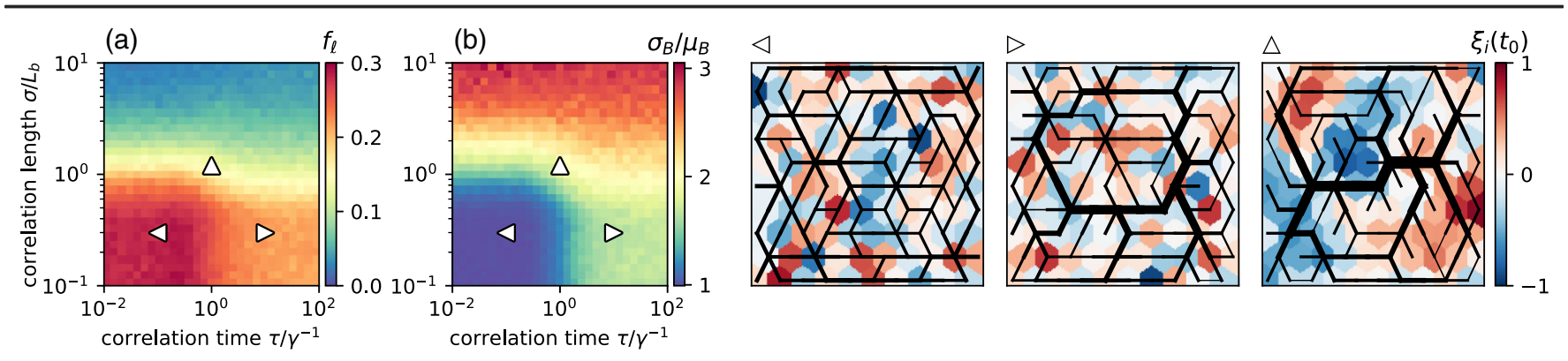

FIG. 3. Combining spatial and temporal correlations leads to three qualitatively distinct NCN phases in the $(\tau, \sigma)$ plane. (a) The loop density $f_{\ell}$ characterizes the three phases as follows: Short correlation times $\tau$ and short correlation lengths $\sigma$ favor highly reticulate redundant networks $(\triangleleft)$, large $\tau$ and small $\sigma$ lead to a moderate reticulation ( $\triangleright$ ), whereas large $\tau$ and large $\sigma$ selects low reticulation $(\triangle)$. (b) The coupling spread $\sigma_{B} / \mu_{B}$ indicates a similar division of the $(\tau, \sigma)$-phase plane: Low $\tau, \sigma$ lead to highly uniform networks $(\triangleleft)$, high $\tau$ and low $\sigma$ lead to networks with an intermediate coupling variability ( $\triangleright$ ), and high $\tau, \sigma$ lead to strongly hierarchical networks with large spread in the couplings $B_{i j}(\triangle)$. The three phases are separated approximately by the lines $\tau / \gamma^{-1} \sim 1$ and $\sigma / L_{b} \sim 1$. Each pixel in the $30 \times 30$ plots (a),(b) is an average over 15 optimal networks; $\alpha=0.5, C=1$ in all panels.

coupling through the cost $C$ pushes the NCNs towards the regime lacking solutions of Eq. (2), but causes no significant changes in topology apart from an overall scaling of the couplings, even very close to the transition [Fig. 1(a)]. Simulations of the full nonlinear Eq. (1) on the identified sparse NCNs confirm a significant noise reduction compared to uniform weights, in quantitative agreement with the predictions of the linear model [dashed and solid lines in Fig. 1(b)]. In general, the linear approximation is accurate as long as the noise is small compared to a worst-case uniform distribution $\varepsilon_{i} \in[-\pi, \pi)$ (Supplemental Material [65]). Since the optimal topologies show little dependence on the nonlinear steady state [Fig. 1(a)], it suffices to focus on the synchronized limit $\bar{\delta}_{i}=0$ and $C=1$ when considering correlated noisy inputs in the remainder. The existence of nontrivial optimal NCN topologies even for uncorrelated inputs is remarkable, and may already have practical applications.

Even more interesting hierarchical NCN structures arise when the input noise becomes correlated [Fig. 2]. The optimal couplings settle into nonuniform patterns containing loopy backbone structures with treelike branches, reminiscent of plant [70,71], fungal [72], or animal [73] vasculature [Fig. 2( $\triangle)$ ]. To dissect the effects of correlations, we first consider fluctuating inputs that are still uncorrelated in time $(\tau \rightarrow 0)$ but have a finite correlation length $\sigma>0$. Our numerical analysis shows that the topology of optimal NCNs changes as $\sigma$ is varied relative to the mean edge length $L_{b}$, the latter defining the natural resolution scale for a network. As expected, for $\sigma \ll L_{b}$, we find the same NCN topology as for incoherent inputs [Figs. 1 and 2]. In contrast, when $\sigma$ becomes comparable to or larger than $L_{b}$, the optimal NCNs become significantly sparser for $0<\alpha<1$ [Fig. 2(a)]. This transition is accompanied by the gradual emergence of a hierarchical network structure, reflected by an increased standard deviation $\sigma_{B}$ of the optimal coupling parameters $B_{i j}$ relative to their mean
$\mu_{B}$ [Fig. 2(b)]. Thus, NCNs for spatially correlated white noise develop hierarchical sparse architectures as the correlation length $\sigma$ increases.

These observations can be rationalized by noting that in the limit of large $\sigma$, we have $\hat{R} \sim D$, where $D$ is the matrix of squared Euclidean distances between oscillators. The rank of $D$ is at most the dimension $d$ of the embedding space [74]. Therefore, the objective Eq. (5) becomes equivalent to an average over at most $d$ steady-state inputs. For networks with a single nonfluctuating input, it is known that the optimal topology is a maximally sparse tree [38]. Since $d=2$ in our case, the optimal NCNs are close to such trees. This argument holds for any sufficiently wellbehaved $\hat{R}=f\left(D / \sigma^{2}\right)$ that depends on the node distances via a scale parameter. The emergence of the hierarchical structure follows from the earlier stated fact that couplings become proportional to a mean flow, which in a treelike topology of steady inputs accumulates as the network graph is traversed upstream from a leaf node. Remarkably, for large $\sigma$, the optimal NCNs often exhibit spontaneous symmetry breaking by approximately realizing rooted trees, in which a hierarchical backbone emanates from one or two central nodes [Fig. 2( $\triangle$ )] even though no such distinguished node(s) were initially prescribed.

Interestingly, colored noise with nonvanishing correlation time $\tau>0$ but no spatial coherence $(\sigma \rightarrow 0)$ has qualitatively similar effects on the network structure. When $\tau$ is larger than the damping timescale $\gamma^{-1}$, optimal NCNs also become sparser and more hierarchically patterned [Figs. 2(c),2(d) and $(\triangleleft, \triangleright)$ ]. The origin of sparsity is now different because $\hat{R}$ is almost full rank for $\sigma \rightarrow 0$, and related to the large- $\tau$ asymptotic behavior of the objective, $\left\langle|\boldsymbol{\varepsilon}(t)|^{2}\right\rangle \sim \operatorname{tr}\left[\left(L^{\dagger}\right)^{2} \hat{R}\right] /(2 \tau)$. Although the objective does not scale homogeneously with $C$ anymore, only the transition between the different $\mathrm{NCN}$ topologies changes (Supplemental Material [65]). 
Lastly, combining spatial and temporal correlations, the $(\tau, \sigma)$ plane subdivides into three distinct phases [Figs. 3(a), 3(b)]. For $\sigma \ll L_{b}$ and $\tau \ll \gamma^{-1}$, optimal NCNs are highly dense and uniform [Fig. 3( $\triangleleft)$ ]. For $\sigma \ll L_{b}$ but $\tau \gg \gamma^{-1}$, NCNs exhibit intermediate sparsity and hierarchical patterning [Fig. 3( $\triangleright$ )]. For $\sigma \gg L_{b}$, NCNs become generally sparse and hierarchically patterned with little dependence on $\tau[$ Fig. 3( $\triangle)$ ], although the transition between the different NCNs topologies is shifted to smaller $\sigma$ when $\tau \gg \gamma^{-1}$.

To conclude, the above analytical and numerical results show that noise cancellation can be hard wired into weighted network topology for both uncorrelated and correlated input fluctuations. As a general rule, the more correlated the input fluctuations, the sparser and more hierarchically ordered the optimal networks become. Previous work $[1,51]$ has demonstrated the applicability of the underlying phase oscillator framework to a myriad of physical and biological systems, from neuronal networks [56,75] and ciliary carpets [76-78] to renewable energy farms and power grids $[9,12,14,50]$. One can therefore expect that the above ideas and results have conceptual and practical implications for most, if not all, of these systems.

This work was supported by an Edmund F. Kelly Research Award (J. D.) and a James S. McDonnell Foundation Complex Systems Scholar Award (J. D.) and the Max Planck Society (M. W.).

*henrikr@mit.edu

†dunkel@mit.edu

*michael.wilczek@ds.mpg.de

[1] B. Lindner, J. Garca-Ojalvo, A. Neiman, and L. SchimanskyGeier, Phys. Rep. 392, 321 (2004).

[2] W. Bialek, Annu. Rev. Biophys. Biophys. Chem. 16, 455 (1987).

[3] L. S. Tsimring, Rep. Prog. Phys. 77, 026601 (2014).

[4] J. Wyngaard, Turbulence in the Atmosphere (Cambridge University Press, Cambridge, England, 2010).

[5] P. Milan, M. Wächter, and J. Peinke, Phys. Rev. Lett. 110, 138701 (2013).

[6] A. Wootten and A. R. Thompson, Proc. IEEE 97, 1463 (2009).

[7] P. Sørensen, A. D. Hansen, and P. A. C. Rosas, J. Wind Eng. Ind. Aerodyn. 90, 1381 (2002).

[8] C. Luo, H. Banakar, B. Shen, and B.-T. Ooi, IEEE Trans. Energy Convers. 22, 341 (2007).

[9] L. J. Lukassen, R. J. A. M. Stevens, C. Meneveau, and M. Wilczek, Wind Energy 21, 474 (2018).

[10] Z. Chen, in Transmission and Distribution Conference and Exhibition, Asia and Pacific, 2005 IEEE/PES (IEEE, New York, 2005), p. 1, doi: 10.1109/TDC.2005.1547160.

[11] J. O. G. Tande, Wind Energy 6, 281 (2003).

[12] K. Schmietendorf, J. Peinke, and O. Kamps, Eur. Phys. J. B 90, 222 (2017).
[13] B. Schäfer, C. Beck, K. Aihara, D. Witthaut, and M. Timme, Nat. Energy 3, 119 (2018).

[14] T. Nesti, A. Zocca, and B. Zwart, Phys. Rev. Lett. 120, 258301 (2018).

[15] H. Haehne, J. Schottler, M. Waechter, J. Peinke, and O. Kamps, Europhys. Lett. 121, 30001 (2018).

[16] T. Coletta, B. Bamieh, and P. Jacquod, Proceedings of CDC '18 (to be published).

[17] D. D. Ridder, S. Vanneste, N. Weisz, A. Londero, W. Schlee, A. B. Elgoyhen, and B. Langguth, Neurosci. Biobehav. Rev. 44, 16 (2014).

[18] A. M. Leaver, L. Renier, M. A. Chevillet, S. Morgan, H. J. Kim, and J. P. Rauschecker, Neuron 69, 33 (2011).

[19] B. B. Averbeck, P. E. Latham, and A. Pouget, Nat. Rev. Neurosci. 7, 358 (2006).

[20] I. Kanitscheider, R. Coen-Cagli, and A. Pouget, Proc. Natl. Acad. Sci. U.S.A. 112, E6973 (2015).

[21] A. Hilfinger and J. Paulsson, Proc. Natl. Acad. Sci. U.S.A. 108, 12167 (2011).

[22] M. Thattai and A. Van Oudenaarden, Proc. Natl. Acad. Sci. U.S.A. 98, 8614 (2001).

[23] J. Paulsson, Nature (London) 427, 415 (2004).

[24] L. O. Chua and L. Yang, IEEE Circuits Syst. Mag. 35, 1273 (1988).

[25] S. Moon and J.-N. Hwang, in Acoustics, Speech, and Signal Processing, 1993, ICASSP-93, 1993 IEEE International Conference on Vol. 1 (IEEE, New York, 1993), p. 573, doi: 10.1109/ICASSP.1993.319183.

[26] I. Lestas, G. Vinnicombe, and J. Paulsson, Nature (London) 467, 174 (2010).

[27] B. Li and K. Y. Michael Wong, Phys. Rev. E 95, 012207 (2017).

[28] M. Tyloo, T. Coletta, and P. Jacquod, Phys. Rev. Lett. 120, 084101 (2018)

[29] C. Zechner, G. Seelig, M. Rullan, and M. Khammash, Proc. Natl. Acad. Sci. U.S.A. 113, 4729 (2016).

[30] R. Bucy, J. Math. Anal. Appl. 20, 1 (1967).

[31] B. Widrow, J. Glover, J. McCool, J. Kaunitz, C. Williams, R. Hearn, J. Zeidler, J. Eugene Dong, and R. Goodlin, Proc. IEEE 63, 1692 (1975).

[32] P. M. Clarkson, Optimal and Adaptive Signal Processing, 1st ed. (CRC Press, New York, 1993).

[33] I. Klickstein, A. Shirin, and F. Sorrentino, Nat. Commun. 8, 15145 (2017)

[34] G. Yan, G. Tsekenis, B. Barzel, J.-J. Slotine, Y.-Y. Liu, and A.-L. Barabási, Nat. Phys. 11, 779 (2015).

[35] Y.-D. Xiao, S.-Y. Lao, L.-L. Hou, and L. Bai, Phys. Rev. E 90, 042804 (2014).

[36] M. Liang, S. Jin, D. Wang, and X. Zou, Eur. Phys. J. B 89, 186 (2016).

[37] W.-X. Wang, X. Ni, Y.-C. Lai, and C. Grebogi, Phys. Rev. E 85, 026115 (2012).

[38] J. R. Banavar, F. Colaiori, A. Flammini, A. Maritan, and A. Rinaldo, Phys. Rev. Lett. 84, 4745 (2000).

[39] E. Katifori, G. J. Szöllősi, and M. O. Magnasco, Phys. Rev. Lett. 104, 048704 (2010).

[40] S. Bohn and M. O. Magnasco, Phys. Rev. Lett. 98, 088702 (2007).

[41] D. Segrè, D. Vitkup, and G. M. Church, Proc. Natl. Acad. Sci. U.S.A. 99, 15112 (2002). 
[42] M. Durand, Phys. Rev. E 73, 016116 (2006).

[43] B. W. Andrews, T.-M. Yi, and P. A. Iglesias, PLoS Comput. Biol. 2, e154 (2006).

[44] E. Kenig, M. C. Cross, R. Lifshitz, R. B. Karabalin, L. G. Villanueva, M. H. Matheny, and M. L. Roukes, Phys. Rev. Lett. 108, 264102 (2012).

[45] P. Ala-Laurila, M. Greschner, E. J. Chichilnisky, and F. Rieke, Nat. Neurosci. 14, 1309 (2011).

[46] B. Schäfer, M. Matthiae, X. Zhang, M. Rohden, M. Timme, and D. Witthaut, Phys. Rev. E 95, 060203 (2017).

[47] K. Schmietendorf, J. Peinke, R. Friedrich, and O. Kamps, Eur. Phys. J. Spec. Top. 223, 2577 (2014).

[48] D. Manik, M. Rohden, H. Ronellenfitsch, X. Zhang, S. Hallerberg, D. Witthaut, and M. Timme, Phys. Rev. E 95, 012319 (2017).

[49] M. M. Bandi, Phys. Rev. Lett. 118, 028301 (2017).

[50] P. H. Nardelli, N. Rubido, C. Wang, M. S. Baptista, C. Pomalaza-Raez, P. Cardieri, and M. Latva-aho, Eur. Phys. J. Spec. Top. 223, 2423 (2014).

[51] J. A. Acebrón, L. L. Bonilla, C. J. Pérez Vicente, F. Ritort, and R. Spigler, Rev. Mod. Phys. 77, 137 (2005).

[52] J. Kromer, A. Khaledi-Nasab, L. Schimansky-Geier, and A. B. Neiman, Sci. Rep. 7, 3956 (2017).

[53] P. Hänggi and P. Jung, Adv. Chem. Phys. 89, 239 (1994).

[54] F. A. Rodrigues, T. K. Peron, P. Ji, and J. Kurths, Phys. Rep. 610, 1 (2016).

[55] B. C. Bag, K. G. Petrosyan, and C.-K. Hu, Phys. Rev. E 76, 056210 (2007).

[56] C. M. Gray, J. Comput. Neurosci. 1, 11 (1994).

[57] T. Tanaka and T. Aoyagi, Phys. Rev. E 78, 046210 (2008).

[58] M. Fazlyab, F. Dörfler, and V. M. Preciado, Automatica 84, 181 (2017).

[59] P. S. Skardal, D. Taylor, and J. Sun, Phys. Rev. Lett. 113, 144101 (2014).

[60] M. Fardad, F. Lin, and M. R. Jovanovic, IEEE Trans. Autom. Control 59, 2457 (2014).
[61] D. Kelly and G. A. Gottwald, Chaos 21, 025110 (2011).

[62] M. Brede, Phys. Lett. A 372, 2618 (2008).

[63] J. H. Meng and H. Riecke, Sci. Rep. 8, 6949 (2018).

[64] D. Hu and D. Cai, Phys. Rev. Lett. 111, 138701 (2013).

[65] See Supplemental Material http://link.aps.org/supplemental/ 10.1103/PhysRevLett.121.208301 for detailed derivations, connection to the Kuramoto model, detailed description of the numerical methods, analysis of noise-canceling improvement, validity of the linear model, dependence of the topology on correlation time, and analysis of square grid topologies.

[66] H. Ronellenfitsch and E. Katifori, Phys. Rev. Lett. 117, 138301 (2016).

[67] A. J. Hudspeth, Neuron 59, 530 (2008).

[68] K. Drescher, R. E. Goldstein, and I. Tuval, Proc. Natl. Acad. Sci. U.S.A. 107, 11171 (2010).

[69] R. J. A. M. Stevens and C. Meneveau, Annu. Rev. Fluid Mech. 49, 311 (2017).

[70] L. Sack and C. Scoffoni, New Phytol. 198, 983 (2013).

[71] H. Ronellenfitsch, J. Lasser, D. C. Daly, and E. Katifori, PLoS Comput. Biol. 11, e1004680 (2015).

[72] L. Heaton, B. Obara, V. Grau, N. Jones, T. Nakagaki, L. Boddy, and M. D. Fricker, Fungal Biol. Rev. 26, 12 (2012).

[73] M. Fruttiger, Invest. Ophthalmol. Visual Sci. 43, 522 (2002).

[74] I. Dokmanic, R. Parhizkar, J. Ranieri, and M. Vetterli, IEEE Signal Process. Mag. 32, 12 (2015).

[75] Y. Penn, M. Segal, and E. Moses, Proc. Natl. Acad. Sci. U.S.A. 113, 3341 (2016).

[76] T. Niedermayer, B. Eckhardt, and P. Lenz, Chaos 18, 037128 (2008).

[77] N. Uchida and R. Golestanian, Europhys. Lett. 89, 50011 (2010).

[78] D. R. Brumley, M. Polin, T. J. Pedley, and R. E. Goldstein, Phys. Rev. Lett. 109, 268102 (2012). 